\title{
EDITORIAL
}

\section{Cellular \& Molecular Immunology receives its first Impact Factor}

\author{
Cellular \& Molecular Immunology (2010) 7, 327; doi:10.1038/cmi.222
}

$\mathrm{T}$ he 2009 edition of the JCR, in which Cellular \& Molecular Immunology (CMI) is listed for the first time, reveals an Impact Factor of 2.765, placing CMI 63rd out of 128 in the Immunology subject category. According to ISI's ranking this also places the journal 5th out of 114 journals published in China and $23 \mathrm{rd}$ out of 587 journals published in the Asia-Pacific region. This is an excellent first Impact Factor for a young journal like CMI and, together with our publishing partner Nature Publishing Group and the continued support of our readers and authors, we hope to further build on this into the future. We are delighted that CMI can be internationally recognized as important in the field of immunology.

CMI has been included in the National Library of Medicine for Index Medicus/MEDLINE database since July, 2005. We applied for approval by NLM/PubMed in February 2005 after the journal had been going for one year, according to NLM regulations. We then applied for inclusion in the Scientific Citation Index (SCI) database in January 2007. We were delighted that CMI was accepted by SCI in October 2007, and even more excited to receive an Impact Factor of 2.765 in June 2010. So, after running for six-and-a-half years, CMI has reached a new milestone.

CMI first published in January 2004. Earlier in May 2003, the Educating and Publishing Committee of Chinese Society of Immunology (CSI) decided to sponsor an official English-language journal, to be named Cellular \& Molecular Immunology. Professor Kuangyen Chou, the vice president of CSI, was elected as the Editor-in-Chief, and Professor Zhigang Tian serves as the Executive Editor-in-Chief of the journal. The CMI editorial office is affiliated to the Institute of Immunology, University of Science \& Technology of China (USTC), where Professor Tian works as the director. USTC and CSI financially sponsor CMI. After six years' successful leadership by Professor Chou, a new international Editorial Board was announced in January 2010. Professor Xuetao Cao from Secondary Millinery
Medical University, China, and Professor Yongjun Liu from MD Anderson Cancer Center, USA, were elected as Co-Editors-in-Chief, and Professor Tian as Deputy Editor-in-Chief. I believe CMI's new era is coming.

Also in January 2010, CMI joined Nature Publishing Group's (NPG's) growing list of high-quality journals. Based on the agreement between CSI, USTC and NPG, CMI have introduced new work flow practices, including new copyediting and typesetting services, and a peer-review tracking system to allow the quickest and most robust manuscript turnaround possible. We are also working with NPG to deliver the journal to the widest audience through hosting on nature. com, abstracting and indexing all content in PubMed, Thomson Reuters ISI and other large abstracting databases, and providing web services such as electronic table of contents alerts, RSS feeds and special web focuses. Through these avenues and through continued development into the future we are working to present the broadest range of the best immunological research to our readership.

Obtaining a first Impact Factor of 2.765 is an excellent result for the authors, readers, and staff of CMI. While we recognize that Impact Factor is not the only way to rate quality, it does reflect our goal of growing and developing CMI through pursuing the highest editorial and scientific standards. These efforts are nothing without the input of the all people who care about and support CMI. Here, we would like to give our heartfelt thanks to the section editors, board members and editorial staff for their endeavor to improve the journal. And of course we thank all the authors and reviewers who have submitted their articles and comments to CMI. The quality of a journal ultimately depends on the manuscripts it publishes and so we hope that our Impact Factor will encourage more and more scientists in immunology to contribute their best research to CMI.

Zhigang Tian, MD, PhD Deputy Editor-in-Chief 